\title{
Chiral Perturbation Theory for Vector Mesons
}

\author{
Elizabeth Jenkins and Aneesh V. Manohar \\ Department of Physics, University of California at San Diego, 9500 Gilman Drive, La Jolla, California 92093-0319
}

Mark B. Wise

California Institute of Technology, Pasadena, California 91125

(Received 19 June 1995)

\begin{abstract}
We derive a heavy vector-meson chiral Lagrangian in which the vector mesons are treated as heavy static matter fields. The unknown couplings of the chiral Lagrangian are further related using the $1 / N_{c}$ expansion. Chiral perturbation theory is applied to the vector-meson mass matrix. At one loop there are large corrections to the individual vector-meson masses, but the singlet-octet mixing angle remains almost unchanged. The parity-violating $s$-wave $\phi \rightarrow \rho \pi$ weak decay amplitude is derived in the combined chiral and large $N_{c}$ limits. Rare $\phi$ decays provide a sensitive test of nonleptonic neutral current structure.
\end{abstract}

PACS numbers: 12.39.Fe, 11.15.Pg, 13.25.Gv, 14.40.Cs

An important application of chiral perturbation theory is to describe the interactions of matter fields (such as nucleons [1] or hadrons containing a heavy quark [2]) with low-momentum pseudo Goldstone bosonsthe pions, kaons and, eta. In this paper we use chiral perturbation theory to describe the interactions of the $\rho$, $K^{*}, \phi$, and $\omega$ vector mesons with low-momentum pseudo Goldstone bosons. The results of this work are relevant for experiments at the $\phi$ factory being built at Frascati [3] We apply chiral perturbation theory to transitions of the form $V \rightarrow V^{\prime} X$, where $V$ and $V^{\prime}$ are vector mesons. The mass differences between the nine lowest-lying vector mesons are small compared with the chiral symmetry breaking scale of $\sim 1 \mathrm{GeV}$, so chiral perturbation theory is applicable as a systematic expansion procedure for such decays. Chiral perturbation theory has previously been used to study processes such as $\rho \rightarrow \pi \pi$, which do not have a vector meson in the final state. Decays such as $\rho \rightarrow \pi \pi$ do not have soft pions in the final state, so the application of the chiral Lagrangian to such processes is not justified and should be considered as a phenomenological model.

The pseudo Goldstone boson fields can be written as a $3 \times 3$ special unitary matrix

$$
\Sigma=\exp \frac{2 i \boldsymbol{\Pi}}{f},
$$

where

$$
\mathbf{I I}=\left[\begin{array}{ccc}
\pi^{0} / \sqrt{2}+\eta / \sqrt{6} & \pi^{+} & K^{+} \\
\pi^{-} & -\pi^{0} / \overline{\sqrt{2}}^{0}+\eta / \sqrt{6} & K^{0} \\
K^{-} & \bar{K}^{0} & -2 \eta / \sqrt{6}
\end{array}\right] .
$$

Under chiral $\mathrm{SU}(3)_{L} \times \mathrm{SU}(3)_{R}, \Sigma \rightarrow L \Sigma R^{\dagger}$, where $L \in$ $\mathrm{SU}(3)_{L}$ and $R \in \mathrm{SU}(3)_{R}$. At leading order in chiral perturbation theory, $f$ can be identified with the pion or kaon decay constant $\left(f_{\pi} \simeq 132 \mathrm{MeV}, f_{K} \simeq 160 \mathrm{MeV}\right)$. It is convenient when describing the interactions of the pseudo Goldstone bosons with other fields to introduce

$$
\xi=\exp \frac{i \boldsymbol{\Pi}}{f}=\sqrt{\Sigma} .
$$

Under chiral $\mathrm{SU}(3)_{L} \times \mathrm{SU}(3)_{R}$,

$$
\xi \rightarrow L \xi U^{\dagger}=U \xi R^{\dagger},
$$

where, in general, $U$ is a complicated function of $L, R$, and the meson fields II. For transformations $V=L=R$ in the unbroken $\mathrm{SU}(3)_{V}$ subgroup, $U=V$.

The vector-meson fields are introduced as a $3 \times 3$ octet matrix

$$
\mathcal{O}_{\mu}=\left[\begin{array}{ccc}
\rho_{\mu}^{0} / \sqrt{2}+\phi_{\mu}^{(8)} / \sqrt{6} & \rho_{\mu}^{+} & K_{\mu}^{*+} \\
\rho_{\mu}^{-} & -\rho_{\mu}^{0} / \sqrt{2}+\phi_{\mu}^{(8)} / \sqrt{6} & K_{\mu}^{* 0} \\
K_{\mu}^{*-} & \bar{K}_{\mu}^{* 0} & -2 \phi_{\mu}^{(8)} / \sqrt{6}
\end{array}\right],
$$

and as a singlet

$$
S_{\mu}=\phi_{\mu}^{(0)} .
$$

Under chiral $\mathrm{SU}(3)_{L} \times \mathrm{SU}(3)_{R}$,

$$
\mathcal{O}_{\mu} \rightarrow U \mathcal{O}_{\mu} U^{\dagger}, \quad S_{\mu} \rightarrow S_{\mu},
$$

and under charge conjugation,

$$
\begin{gathered}
C \mathcal{O}_{\mu} C^{-1}=-\mathcal{O}_{\mu}^{T}, \quad C S_{\mu} C^{-1}=-S_{\mu}, \\
C \xi C^{-1}=\xi^{T} .
\end{gathered}
$$

We construct a chiral Lagrangian for vector mesons by treating the vector mesons as heavy static fields

(C) 1995 The American Physical Society 
[4,5] with fixed four-velocity $v^{\mu}, v^{2}=1$. The three polarization states of vector mesons with velocity $v^{\mu}$ satisfy $v \cdot S=v \cdot \mathcal{O}=0$. The chiral Lagrange density which describes the interactions of the vector mesons with the low-momentum $\pi, K$, and $\eta$ mesons has the general structure

$$
\mathcal{L}=\mathcal{L}_{\mathrm{kin}}+\mathcal{L}_{\text {int }}+\mathcal{L}_{\text {mass }} .
$$

Processes such as $\phi \rightarrow K^{+} K^{-}$cause a net disappearance of vector mesons and can be taken into account by including anti-Hermitian terms in $\mathcal{L}$. The vector-meson widths are small compared with $M_{K}$ and will be neglected in our analysis.

At leading order in the derivative and quark mass expansions

$$
\mathcal{L}_{\mathrm{kin}}=-i S_{\mu}^{\dagger}(v \cdot \partial) S^{\mu}-i \operatorname{Tr} \mathcal{O}_{\mu}^{\dagger}(v \cdot \mathcal{D}) \mathcal{O}^{\mu}
$$

and

$$
\begin{aligned}
\mathcal{L}_{\text {int }}= & i g_{1} S_{\mu}^{\dagger} \operatorname{Tr}\left(\mathcal{O}_{\nu} A_{\lambda}\right) v_{\sigma} \epsilon^{\mu \nu \lambda \sigma}+\text { H.c. } \\
& +i g_{2} \operatorname{Tr}\left(\left\{\mathcal{O}_{\mu}^{\dagger}, \mathcal{O}_{\nu}\right\} A_{\lambda}\right) v_{\sigma} \epsilon^{\mu \nu \lambda \sigma},
\end{aligned}
$$

where

$$
\mathcal{D}^{\nu} \mathcal{O}^{\mu}=\partial^{\nu} \mathcal{O}^{\mu}+\left[V^{\nu}, \mathcal{O}^{\mu}\right]
$$

and

$$
\begin{aligned}
V^{\mu} & =\frac{1}{2}\left(\xi \partial^{\mu} \xi^{\dagger}+\xi^{\dagger} \partial^{\mu} \xi\right), \\
A^{\mu} & =\frac{i}{2}\left(\xi \partial^{\mu} \xi^{\dagger}-\xi^{\dagger} \partial^{\mu} \xi\right) .
\end{aligned}
$$

The terms in $\mathcal{L}_{\text {kin }}$ appear with minus signs because the polarization vector is spacelike. Charge conjugation invariance requires that the product of $\mathcal{O}_{\mu}^{\dagger}$ and $\mathcal{O}_{\nu}$ in the second term of Eq. (11) be an anticommutator. (It is important to remember that in the matrix $\mathcal{O}_{\mu}^{\dagger}$ the field $\rho_{\mu}^{-\dagger}$ is not equal to $\rho_{\mu}^{+}$, etc. In heavy vector-meson chiral perturbation theory, $\rho_{\mu}^{+}$destroys a $\rho^{+}$but it does not create the corresponding antiparticle. A separate field $\rho_{\mu}^{-\dagger}$ is introduced to create a $\rho^{-}$.) Finally, to linear order in the quark mass expansion,

$$
\begin{aligned}
\mathcal{L}_{\text {mass }}= & \mu_{0} S_{\mu}^{\dagger} S^{\mu}+\mu_{8} \operatorname{Tr} \mathcal{O}_{\mu}^{\dagger} \mathcal{O}^{\mu} \\
& +\lambda_{1}\left[\operatorname{Tr}\left(\mathcal{O}_{\mu}^{\dagger} \mathcal{M}_{\xi}\right) S^{\mu}+\text { H.c. }\right] \\
& +\lambda_{2} \operatorname{Tr}\left(\left\{\mathcal{O}_{\mu}^{\dagger}, \mathcal{O}^{\mu}\right\} \mathcal{M}_{\xi}\right) \\
& +\sigma_{0} \operatorname{Tr} \mathcal{M}_{\xi} S_{\mu}^{\dagger} S^{\mu}+\sigma_{8} \operatorname{Tr} \mathcal{M}_{\xi} \operatorname{Tr} \mathcal{O}_{\mu}^{\dagger} \mathcal{O}^{\mu},
\end{aligned}
$$

where $\mathcal{M}$ is the quark mass matrix $\mathcal{M}=$ $\operatorname{diag}\left(m_{u}, m_{d}, m_{s}\right)$ and

$$
\mathcal{M}_{\xi}=\frac{1}{2}\left(\xi \mathcal{M} \xi+\xi^{\dagger} \mathcal{M} \xi^{\dagger}\right)
$$

Note that the fields $S$ and $\mathcal{O}$ appearing in Eqs. (10)(12) are understood to be velocity-dependent fields which are rescaled by a common phase factor (ei- ther $e^{-i \mu_{0} v \cdot x}$ or $e^{-i \mu_{8} v \cdot x}$ ). (The velocity-dependent vector-meson fields are related to the vector-meson fields by $\phi_{v}^{\mu}=\sqrt{2 m} e^{i m v \cdot x} \phi^{\mu}$ and have dimension $3 / 2$.) This rescaling removes either $\mu_{0}$ or $\mu_{8}$ from Eq. (14), so only the singlet-octet mass difference $\Delta \mu \equiv \mu_{0}-\mu_{8}$ is relevant. Phenomenologically, the parameter $\Delta \mu<200 \mathrm{MeV}$ is comparable to mass splittings of order $m_{s}$, so in our power counting we treat $\Delta \mu$ as a quantity of order $m_{q} . \Delta \mu$ is of order $1 / N_{c}$, and so vanishes in the large $N_{c}$ limit.

We begin by considering the spectrum of vector mesons produced at leading order in chiral perturbation theory. The analysis is identical to the well-known $\mathrm{SU}(3)$ analysis [6]. Neglecting isospin breaking due to the up and down quark mass difference, i.e., $m_{u}=m_{d}=\hat{m}$, we find that

$$
m_{\rho}=\bar{\mu}_{8}+2 \lambda_{2} \hat{m}, \quad m_{K^{*}}=\bar{\mu}_{8}+\lambda_{2}\left(\hat{m}+m_{s}\right),
$$

and the $\phi^{(0)}-\phi^{(8)}$ mass matrix is

$$
M^{(08)}=\left[\begin{array}{cc}
\bar{\mu}_{0} & -\frac{2}{\sqrt{6}} \lambda_{1}\left(m_{s}-\hat{m}\right) \\
-\frac{2}{\sqrt{6}} \lambda_{1}\left(m_{s}-\hat{m}\right) & \bar{\mu}_{8}+\frac{2}{3} \lambda_{2}\left(\hat{m}+2 m_{s}\right)
\end{array}\right],
$$

where

$$
\bar{\mu}_{0}=\mu_{0}+\sigma_{0} \operatorname{Tr} \mathcal{M}, \quad \bar{\mu}_{8}=\mu_{8}+\sigma_{8} \operatorname{Tr} \mathcal{M} .
$$

(Isospin breaking effects have been studied in Ref. [7].) Using Eqs. (16a) and (16b), it is possible to express the elements of $M^{(08)}$ in terms of the measured vector-meson masses (up to a sign ambiguity for $M_{12}^{(08)}$ )

$$
\begin{aligned}
M_{11}^{(08)}= & m_{\omega}+m_{\phi}-\frac{4}{3} m_{K^{*}}+\frac{1}{3} m_{\rho}, \\
M_{22}^{(08)}= & \frac{4}{3} m_{K^{*}}-\frac{1}{3} m_{\rho}, \\
M_{12}^{(08)}= & M_{21}^{(08)}= \pm\left[\left(\frac{4}{3} m_{K^{*}}-\frac{1}{3} m_{\rho}-m_{\omega}\right)\right. \\
& \left.\times\left(m_{\phi}-\frac{4}{3} m_{K^{*}}+\frac{1}{3} m_{\rho}\right)\right]^{1 / 2} .
\end{aligned}
$$

The eigenstates of $M^{(08)}$ are parametrized by a mixing angle $\Theta_{V}$

$$
\begin{aligned}
& |\phi\rangle=\sin \Theta_{V}\left|\phi^{(0)}\right\rangle-\cos \Theta_{V}\left|\phi^{(8)}\right\rangle, \\
& |\omega\rangle=\cos \Theta_{V}\left|\phi^{(0)}\right\rangle+\sin \Theta_{V}\left|\phi^{(8)}\right\rangle,
\end{aligned}
$$

where Eqs. (18) imply the usual $\mathrm{SU}(3)_{V}$ prediction for the tangent of the mixing angle

$$
\tan \Theta_{V}=\mp \sqrt{\frac{m_{\phi}-\frac{4}{3} m_{K^{*}}+\frac{1}{3} m_{\rho}}{\frac{4}{3} m_{K^{*}}-\frac{1}{3} m_{\rho}-m_{\omega}}} \simeq \mp 0.76 .
$$

In the large $N_{c}$ limit [8,9], quark loops are suppressed so that the leading diagrams in the meson sector contain a single quark loop. As a result, the octet and singlet mesons can be combined into a single "nonet" matrix

$$
N_{\mu}=\mathcal{O}_{\mu}+\frac{I}{\sqrt{3}} S_{\mu}
$$


which enters the chiral Lagrangian. The kinetic, interaction, and mass terms at leading order in $1 / N_{c}$ are

$$
\begin{gathered}
\mathcal{L}_{\mathrm{kin}} \rightarrow-i \operatorname{Tr} N_{\mu}^{\dagger}(v \cdot \mathcal{D}) N^{\mu}, \\
\mathcal{L}_{\mathrm{int}} \rightarrow i g_{2} \operatorname{Tr}\left(\left\{N_{\mu}^{\dagger}, N_{\nu}\right\} A_{\lambda}\right) v_{\sigma} \epsilon^{\mu \nu \lambda \sigma},
\end{gathered}
$$

and

$$
\mathcal{L}_{\text {mass }} \rightarrow \mu \operatorname{Tr} N_{\mu}^{\dagger} N^{\mu}+\lambda_{2} \operatorname{Tr}\left(\left\{N_{\mu}^{\dagger}, N^{\mu}\right\} \mathcal{M}_{\xi}\right) .
$$

Comparing with Eqs. (10)-(14), one finds that, in the $N_{c} \rightarrow \infty$ limit,

$$
\begin{aligned}
\Delta \mu \rightarrow 0, \quad \sigma_{0} \rightarrow \frac{2 \lambda_{2}}{3}, \quad \sigma_{8} & \rightarrow 0, \\
g_{1} \rightarrow \frac{2 g_{2}}{\sqrt{3}}, \quad \lambda_{1} & \rightarrow \frac{2 \lambda_{2}}{\sqrt{3}}, \quad \tan \Theta_{V} \rightarrow \frac{1}{\sqrt{2}},
\end{aligned}
$$

the $|\phi\rangle$ state becomes "pure" $|s \bar{s}\rangle$, and the nonet matrix is

$$
N_{\mu}=\left[\begin{array}{ccc}
\rho_{\mu}^{0} / \sqrt{2}+\omega_{\mu} / \sqrt{2} & \rho_{\mu}^{+} & K_{\mu}^{*+} \\
\rho_{\mu}^{-} & -\rho_{\mu}^{0} / \sqrt{2}^{-}+\omega_{\mu} / \sqrt{2} & K_{\mu}^{* 0} \\
K_{\mu}^{*-} & \bar{K}_{\mu}^{* 0} & \phi_{\mu}
\end{array}\right] .
$$

If the minus sign is chosen in Eq. (18c), the prediction for the mixing angle at leading order in chiral perturbation theory, Eq. (20), is close to its value for large $N_{c}$. At leading order in chiral perturbation theory, the partial width for the Zweig forbidden decay $\phi \rightarrow \rho \pi$ summed over all three modes is

$$
\Gamma(\phi \rightarrow \rho \pi)=\frac{2 h^{2}\left|\boldsymbol{p}_{\pi}\right|^{3}}{\pi f^{2}} .
$$

The coupling, $h$, which vanishes as $N_{c} \rightarrow \infty$, is

$$
h=\frac{g_{1}}{\sqrt{2}} \sin \Theta_{V}-\frac{g_{2}}{\sqrt{3}} \cos \Theta_{V} .
$$

The measured branching ratio gives $h \approx 0.05$, which also suggests that the couplings are close to the $N_{c} \rightarrow \infty$ values.

In the nonrelativistic constituent quark model, assuming the $|\phi\rangle$ is pure $|s \bar{s}\rangle, g_{1}=2 / \sqrt{3}$ and $g_{2}=1$. In the nonrelativistic chiral quark model [10], $g_{1}$ and $g_{2}$ are reduced by a factor of 0.75 from their values in the nonrelativistic constituent quark model.

In chiral perturbation theory the leading corrections to the expressions for the vector-meson masses in Eqs. (16) are of order $m_{q}^{3 / 2}$ (recall we are treating $\Delta \mu$ as of order $\left.m_{q}\right)$ and arise from one-loop self-energy diagrams giving

$$
\begin{aligned}
\delta m_{\rho} & =-\frac{1}{12 \pi f^{2}}\left[g_{2}^{2}\left(\frac{2}{3} m_{\pi}^{3}+2 m_{K}^{3}+\frac{2}{3} m_{\eta}^{3}\right)+g_{1}^{2} m_{\pi}^{3}\right], \\
\delta m_{K^{*}} & =-\frac{1}{12 \pi f^{2}}\left[g_{2}^{2}\left(\frac{3}{2} m_{\pi}^{3}+\frac{5}{3} m_{K}^{3}+\frac{1}{6} m_{\eta}^{3}\right)+g_{1}^{2} m_{K}^{3}\right], \\
\delta M_{11}^{(08)} & =-\frac{1}{12 \pi f^{2}} g_{1}^{2}\left(3 m_{\pi}^{3}+4 m_{K}^{3}+m_{\eta}^{3}\right), \\
\delta M_{22}^{(08)} & =-\frac{1}{12 \pi f^{2}}\left[g_{2}^{2}\left(2 m_{\pi}^{3}+\frac{2}{3} m_{K}^{3}+\frac{2}{3} m_{\eta}^{3}\right)+g_{1}^{2} m_{\eta}^{3}\right], \\
\delta M_{12}^{(08)} & =\delta M_{21}^{(08)}=\frac{1}{12 \pi f^{2}} \sqrt{\frac{2}{3}} g_{1} g_{2}\left(-3 m_{\pi}^{3}+2 m_{K}^{3}+m_{\eta}^{3}\right) .
\end{aligned}
$$

The singlet-octet mixing angle $\Theta_{V}$ including these corrections is

$$
\tan \Theta_{V}=\mp \sqrt{\frac{m_{\phi}-\frac{4}{3} m_{K^{*}}+\frac{1}{3} m_{\rho}-\delta m}{\frac{4}{3} m_{K^{*}}-\frac{1}{3} m_{\rho}-m_{\omega}+\delta m}},
$$

where

$$
\begin{aligned}
\delta m & =-\frac{4}{3} \delta m_{K^{*}}+\frac{1}{3} \delta m_{\rho}+\delta M_{22}^{(08)} \\
& =-\frac{1}{12 \pi f^{2}}\left(g_{1}^{2}+\frac{2}{3} g_{2}^{2}\right)\left(\frac{1}{3} m_{\pi}^{3}-\frac{4}{3} m_{K}^{3}+m_{\eta}^{3}\right) .
\end{aligned}
$$

Using the relation between $g_{2}$ and $g_{1}$ in Eq. (26), we find that

$$
\delta m \rightarrow-\frac{2 g_{2}^{2}}{12 \pi f^{2}}\left(\frac{1}{3} m_{\pi}^{3}-\frac{4}{3} m_{K}^{3}+m_{\eta}^{3}\right) .
$$

With $g_{2}=0.75$, Eq. (33) yields $\delta m \simeq-4 \mathrm{MeV}$. The combination of mass shifts, $\delta m$, that affects the mixing angle $\Theta_{V}$ is very small even though the corrections to the individual masses are substantial (e.g., $\delta m_{\rho} \simeq-300$ $\mathrm{MeV}) . \delta m$, which is of order $1 / N_{c}$, must transform like a 27 of flavor SU(3). The linear combination of the cubed pseudoscalar meson mass in Eq. (33) transforms like a 27, and is numerically small. This same linear combination occurs in the violation of the Gell-Mann-Okubo formula for baryon masses [11].

For $N_{c}$ large, the $\phi \rightarrow \rho \pi$ decay amplitude is of order $N_{c}^{-3 / 2}$, since the leading order $1 / \sqrt{N_{c}}$ amplitude is forbidden by Zweig's rule. At leading order in chiral perturbation theory it occurs at tree level because of order $1 / N_{c}$ deviations from the relations $\tan \Theta_{V}=1 / \sqrt{2}$ and $g_{1} / g_{2}=2 / \sqrt{3}$. At order $m_{s} \ln m_{s}$ in the chiral expansion the order $N_{c}^{-3 / 2}$ contribution arises from one-loop vertex and wave function corrections calculated with vertices from the nonet Lagrange density. The $\pi$ and $\eta$ loops do not contribute when one uses the nonet Lagrangian. The resulting decay amplitude for each of the three $\rho \pi$ modes is

$$
\begin{aligned}
\mathcal{A}(\phi \rightarrow \rho \pi)= & \frac{i}{f} \epsilon^{\mu \nu \lambda \sigma} \epsilon_{\mu}(\phi) \epsilon_{\nu}^{*}(\rho) p_{\pi \lambda} v_{\sigma}\left(2 \sqrt{m_{\phi} m_{\rho}}\right) \\
& \times\left[\sqrt{2} h-g_{2}^{3}\left(\frac{m_{K}^{2}}{8 \pi^{2} f^{2}}\right) \ln \left(\frac{m_{K}^{2}}{\mu^{2}}\right)+\cdots\right],
\end{aligned}
$$

where the ellipsis denotes terms of higher order in the chiral and $1 / N_{c}$ expansions. The terms of order $m_{s}$ have a dependence on the subtraction point $\mu$ which cancels that of the logarithm in Eq. (34). With $\mu=1 \mathrm{GeV}$, $g_{2}=0.75$, the magnitude of the term of order $m_{s} \ln m_{s}$ in Eq. (34) is about 1.5 times as large as the measured $\phi \rightarrow \rho \pi$ decay amplitude. This suggests either that $g_{2}$ is smaller than the chiral quark model value or that there 
is a partial cancellation between order $m_{s} \ln m_{s}$ and order $m_{s}$ contributions to the decay amplitude.

The Frascati $\phi$ factory is expected to produce of order $10^{10} \phi$ 's, allowing even very rare $\phi$ decay processes to be experimentally accessible. The $\phi \rightarrow \rho \pi$ decay amplitude has a small parity-violating $s$-wave amplitude that is induced by the weak interactions. This amplitude can be predicted in the combined limits of chiral $\mathrm{SU}(2)_{L} \times \mathrm{SU}(2)_{R}$ symmetry and large $N_{c}$. In these limits the part of the weak Hamiltonian that dominates the $s$-wave $\phi \rightarrow \rho \pi$ amplitude is due to $Z^{0}$ exchange,

$$
\begin{aligned}
\mathcal{H}_{W}= & \eta \frac{G_{F}}{2 \sqrt{2}}\left(1-\frac{4}{3} \sin ^{2} \theta_{W}\right) \\
& \times\left(\bar{s}_{\alpha} \gamma_{\mu} s_{\alpha}\right)\left[\bar{u}_{\beta} \gamma^{\mu} \gamma_{5} u_{\beta}-\bar{d}_{\beta} \gamma^{\mu} \gamma_{5} d_{\beta}\right],
\end{aligned}
$$

where $\eta \sim 1.56$ [12] arises from QCD scaling between the weak scale and low energies. In the large $N_{c}$ limit, $\log \eta$ is of order $1 / N_{c}$ times logarithms of the form $\log M_{W} / \Lambda$, and we have chosen to include corrections of this form [13]. The $\phi \rightarrow \rho \pi$ matrix element takes the form

$$
\begin{aligned}
\left\langle\rho \pi\left|\mathcal{H}_{W}\right| \phi\right\rangle= & \eta \frac{G_{F}}{2 \sqrt{2}}\left(1-\frac{4}{3} \sin ^{2} \theta_{W}\right) f_{\phi} \epsilon_{\mu}(\phi) \\
& \times\left\langle\rho \pi\left|\bar{u} \gamma^{\mu} \gamma_{5} u-\bar{d} \gamma^{\mu} \gamma_{5} d\right| 0\right\rangle
\end{aligned}
$$

where the $\phi$ decay constant $f_{\phi}$ is defined by

$$
\left\langle 0\left|\bar{s} \gamma_{\mu} s\right| \phi\right\rangle=f_{\phi} \epsilon_{\mu}(\phi)
$$

The measured $\phi \rightarrow e^{+} e^{-}$decay width implies that $f_{\phi} \simeq(492 \mathrm{MeV})^{2}$. The left-handed isovector current $\bar{u} \gamma^{\mu} P_{L} u-\bar{d} \gamma^{\mu} P_{L} d$ transforms as $\left(3_{L}, 1_{R}\right)$ under chiral $\mathrm{SU}(2)_{L} \times \mathrm{SU}(2)_{R}$. For matrix elements between the vacuum and a $\rho$ plus soft pions, this current is represented by the operator

$$
\bar{u} \gamma^{\mu} P_{L} u-\bar{d} \gamma^{\mu} P_{L} d=\frac{f_{\rho}}{2 \sqrt{2 m_{\rho}}} \operatorname{Tr}\left(\xi \mathcal{O}_{\mu}^{\dagger} \xi^{\dagger} \tau^{3}\right)
$$

where $f_{\rho}$ is defined analogous to $f_{\phi}$ and has the value $f_{\rho} \simeq(407 \mathrm{MeV})^{2}$ from the $\rho \rightarrow e^{+} e^{-}$partial width. In Eq. (38), $\xi$ and $\mathcal{O}_{\mu}$ are the $2 \times 2$ matrix analogs of the corresponding $3 \times 3$ matrices used in the case of chiral $\mathrm{SU}(3)_{L} \times \mathrm{SU}(3)_{R}$. The right-handed isovector current is given by exchanging $\xi$ and $\xi^{\dagger}$ in Eq. (38). Using Eq. (38) to evaluate the matrix element in Eq. (36) we find that in the combined chiral and large $N_{C}$ limits

$$
\begin{aligned}
\left\langle\rho^{+} \pi^{-}\left|\mathcal{H}_{W}\right| \phi\right\rangle_{s \text {-wave }}= & -\left\langle\rho^{-} \pi^{+}\left|\mathcal{H}_{W}\right| \phi\right\rangle_{s \text {-wave }} \\
= & -i \eta \frac{G_{F}}{\sqrt{2}}\left(1-\frac{4}{3} \sin ^{2} \theta_{W}\right) \\
& \times\left(\frac{f_{\phi} f_{\rho}}{f}\right) \epsilon^{*}(\rho) \cdot \epsilon(\phi)
\end{aligned}
$$

and

$$
\left\langle\rho^{0} \pi^{0}\left|\mathcal{H}_{W}\right| \phi\right\rangle_{s \text {-wave }}=0
$$

Interference between the $s$-wave and $p$-wave amplitudes is possible for aligned $\phi$ 's, but it requires a final state interaction phase. The $s$-wave $\rho^{+} \pi^{-}$branching ratio is $10^{-11}$, which is too small to be measured at the Frascati $\phi$ factory. However, an enhancement of the parityviolating decay rate could make the signal observable. This provides a very interesting test of new physics, because it probes nonleptonic neutral currents involving strange quarks. We will consider the application of chiral perturbation theory to other processes such as $\phi \rightarrow \rho \gamma \gamma$ elsewhere.

This work was supported in part by the Department of Energy under Grant No. DOE-FG03-90ER40546 and No. DE-FG03-92-ER40701. E.J. was supported in part by the NYI program, through Grant No. PHY-9457911 from the National Science Foundation, and by the Alfred P. Sloan Foundation. A. M. was supported in part by the PYI program, through Grant No. PHY-8958081 from the National Science Foundation.

[1] J. Gasser, M.E. Sainio, and A. S̆varc, Nucl. Phys. B307, 779 (1988); E. Jenkins and A. V. Manohar, Phys. Lett. B 255, 558 (1991); 259, 353 (1991).

[2] M. B. Wise, Phys. Rev. D 45, 2188 (1992); G. Burdman and J. F. Donoghue, Phys. Lett. B 280, 287 (1992); T.-M. Yan et al., Phys. Rev. D 46, 1148 (1992); P. Cho, Nucl. Phys. B396, 183 (1993).

[3] The DAФNE Physics Handbook, edited by L. Maiani, G. Pancheri, and N. Paver (INFN, Frascati, Italy, 1992).

[4] E. Eichten and B. Hill, Phys. Lett. B 234, 511 (1990).

[5] H. Georgi, Phys. Lett. B 240, 447 (1990).

[6] J. J. J. Kokkedee, The Quark Model (Benjamin, New York, 1969).

[7] R. Urech, Report No. hep-ph/9504238.

[8] G. 't Hooft, Nucl. Phys. B72, 461 (1974).

[9] G. Veneziano, Nucl. Phys. B117, 519 (1976).

[10] A. Manohar and H. Georgi, Nucl. Phys. B234, 189 (1984).

[11] E. Jenkins, Nucl. Phys. B368, 190 (1992).

[12] J. Dai, M. J. Savage, J. Liu, and R. Springer, Phys. Lett. B 271, 403 (1991).

[13] W. Bardeen, A. Buras, and J. M. Gerard, Nucl. Phys. B293, 787 (1987). 